\title{
Multi-Label Multi-Kernel Transfer Learning for Human Protein Subcellular Localization
}

\author{
Suyu Mei ${ }^{*}$
}

Software College, Shenyang Normal University, Shenyang, China

\begin{abstract}
Recent years have witnessed much progress in computational modelling for protein subcellular localization. However, the existing sequence-based predictive models demonstrate moderate or unsatisfactory performance, and the gene ontology (GO) based models may take the risk of performance overestimation for novel proteins. Furthermore, many human proteins have multiple subcellular locations, which renders the computational modelling more complicated. Up to the present, there are far few researches specialized for predicting the subcellular localization of human proteins that may reside in multiple cellular compartments. In this paper, we propose a multi-label multi-kernel transfer learning model for human protein subcellular localization (MLMK-TLM). MLMK-TLM proposes a multi-label confusion matrix, formally formulates three multilabelling performance measures and adapts one-against-all multi-class probabilistic outputs to multi-label learning scenario, based on which to further extends our published work GO-TLM (gene ontology based transfer learning model for protein subcellular localization) and MK-TLM (multi-kernel transfer learning based on Chou's PseAAC formulation for protein submitochondria localization) for multiplex human protein subcellular localization. With the advantages of proper homolog knowledge transfer, comprehensive survey of model performance for novel protein and multi-labelling capability, MLMK$T L M$ will gain more practical applicability. The experiments on human protein benchmark dataset show that MLMK-TLM significantly outperforms the baseline model and demonstrates good multi-labelling ability for novel human proteins. Some findings (predictions) are validated by the latest Swiss-Prot database. The software can be freely downloaded at http://soft. synu.edu.cn/upload/msy.rar.
\end{abstract}

Citation: Mei S (2012) Multi-Label Multi-Kernel Transfer Learning for Human Protein Subcellular Localization. PLoS ONE 7(6): e37716. doi:10.1371/ journal.pone.0037716

Editor: Francisco José Esteban, University of Jaén, Spain

Received September 29, 2011; Accepted April 28, 2012; Published June 13, 2012

Copyright: $\odot 2012$ Suyu Mei. This is an open-access article distributed under the terms of the Creative Commons Attribution License, which permits unrestricted use, distribution, and reproduction in any medium, provided the original author and source are credited.

Funding: The author has no support or funding to report.

Competing Interests: The author has declared that no competing interests exist.

*E-mail: 061021053@fudan.edu.cn

\section{Introduction}

Recent years have witnessed much progress in computational modelling for protein subcellular localization [1]. However, researches on human genome and proteomics seem more urgent and important for human disease diagnosis and drug development. Unfortunately, there are far few specialized predictive models for human protein subcellular localization thus far [2,3,4,5]. Furthermore, many human proteins have multiple subcellular locations, which renders the computational modelling more complicated. Up to the present, there are only two models (Hum-mPLoc [4] and Hum-mPLoc 2.0 [5]) that can be applicable to multiple subcellular localization of human proteins.

Although many protein sequence feature extraction methods have been successfully developed for protein subcellular localization, such as signal peptide [6], sequence domain [7], PSSM [8,9], $k$-mer [10,11] etc., the accuracy of the models is still moderate or unsatisfactory, most of which average about 70\% [6,7,9,10,11]. Garg A et al (2005) [3] used sequence features only (amino acid composition and its order information) for human protein subcellular localization, and the result is satisfactory $(84.9 \%)$, but it covers only 4 subcellular locations. The Gene Ontology $(G O)$ project has developed three structured controlled vocabularies (ontologies) that describe gene products in terms of their associated biological processes, cellular components and molecular functions in a species-independent manner, and the $G O A$ database [12] provides high-quality electronic and manual associations (annotations) of $G O$ terms to UniProt Knowledgebase (UniProtKB) entries [13]. Because the three aspects of gene ontology are closely related and the $G O$ terms of cellular component contains direct indicative information about protein subcellular location, $G O$ has become a generally effective feature for the prediction of protein subcellular localization $[2,4,5,14,15,16,17,18,19,20,21,22,23,24,25,26,27,28,29]$. Chou K.C. et al [2] proposed an ensemble leaning model called Hum$P L o c$ for human protein subcellular localization. The model consists of two parts: GO-based $k N \mathcal{N}$ and PseAAC-based $k N \mathcal{N}$, and the latter part was designed to compensate for the model performance in the case of $G O$ unavailability. To cover multiplex human proteins that reside in or transport across multiple subcellular locations, Shen HB et al [4] further proposed an improved model called Hum-mPLoc, which extended the number of subcellular locations from 12 to 14 and formally formulated the concept of locative protein and the success rate for multiplex protein subcellular localization. Hum-PLoc, Hum-mPLoc and the work $[2,15,16,17,18,19,20,21,22]$ used the target protein's own $G O$ information to train model, thus inapplicable to novel protein prediction. Many recent $G O$-based methods generally exploit the homolog $G O$ information for novel protein subcellular localization $[5,23,24,25,26,27,28,29,30]$. Based on Hum$m P L o c$, Shen HB et al [5] further proposed Hum-mPLoc2.0 for multiplex and novel human protein subcellular localization, where a more stringent human dataset with $25 \%$ sequence similarity 
threshold is constructed to train a $k \mathcal{N N}$ ensemble classifier. Hum$m P L o c 2.0$ incorporated those homologs with sequence similarity $\geq 60 \%$, but achieved relatively low accuracy $(62.7 \%)$. However, the method of setting threshold for homolog incorporation has the following disadvantages: (1) significant homolog (high sequence identity, assuming $\geq 60 \%$ ) may potentially be divergent from the target protein in terms of protein subcellular localization, for instance, the target protein P21291 resides in subcellular locations: Nucleus, while its significant homolog P67966 (sequence identity: 90.16\%; PSI-Blast E-value: 13e-174072, obtained by Blast default options) resides in subcellular locations: Cytoplasm and Cytoskeleton. High threshold of sequence identity, e.g. $60 \%$, can not guarantee that no noise would be introduced to the target protein; (2) remote homolog (low sequence identity, assuming $<30 \%$ ) may be convergent to the target protein in terms of protein subcellular localization, for instance, the target protein $P 21291$ resides in subcellular locations: Endoplasmic reticulum, Membrane and Microsome, while its first 7 significant remote homologs queried against SwissProt 57.3 database [13] with default Blast option: $075881\left(26.82 \%, 4 e^{-041)}, 002766\left(25.05 \%, 4 e^{-028}\right)\right.$, Q63688 (25.66\%,2e-027),P22680(23.68\%,4e-026),Q16850(23.92\%,

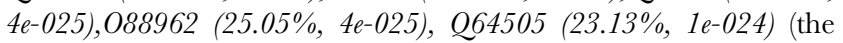
first number in parenthesis denotes sequence identity and the second number denotes PSI-Blast E-value), also reside in the subcellular locations: Endoplasmic reticulum, Membrane and Microsome. High threshold of sequence identity $(60 \%)$ would filter out all the convergent remote homologs that are informative to protein subcellular localization, and thus no homolog knowledge would be transferred to the target protein P21291. We can see that both significant homolog and remote homolog can be convergent homolog, or divergent homolog in terms of protein subcellular localization, thus we should conduct homolog knowledge transfer in a proper way, so that the noise from divergent homolog can be effectively depressed. Mei S et al [25] proposed a transfer learning model (gene ontology based transfer learning for protein subcellular localization, $G O-T L M$ ) to measure the individual contribution of $G O$ three aspects to the model performance, where the kernel weights are evaluated by simple nonparametric cross validation. Mei S [26] further proposed an improved transfer learning model $(M K-T L M)$, which conducted improvements on GO-TLM from the two major concerns: (1) more rational noise control over divergent homolog knowledge transfer; (2) comprehensive survey of model performance, especially for novel protein prediction. However, many human proteins reside in or transport across multiple cellular compartments, and the proteins with multiple locations may help reveal special biological implications to basic research and drug discovery [30,31]. Neither GO-TLM nor $M K-T L M$ is applicable to multiple protein subcellular localization prediction.

In this paper, we propose a multi-label multi-kernel transfer learning model for large-scale human protein subcellular localization $(M L M K-T L M)$. Based on the work [25,26], MLMK-TLM proposes a multi-label confusion matrix and adapts one-against-all multi-class probabilistic outputs to multi-label learning scenario. With the advantages of proper homolog knowledge transfer, comprehensive survey of model performance for novel protein and multi-labelling capability, $M L M K-T L M$ gains more practical applicability. To validate $M L M K-T L M$ 's effectiveness, we conduct a comprehensive model evaluation on the latest human protein dataset Hum-mPLoc 2.0 [5].

\section{Methods}

\section{Transfer learning}

As a research field of machine learning community, transfer learning has attracted more and more attentions in recent years
[32]. Traditional supervised learning generally assumes that all the data, including training data and unseen test data, are subjected to independent and identical distribution (iid), which doesn't hold true under many practical circumstances, especially in the field of biological data analysis. For example, the microarray gene expression data from different experimental platforms would be subjected to different level of experimental noise [33]. Transfer learning can be viewed as a bridge to transfer useful knowledge across two related domains with heterogeneous feature representations and different distributions. Pan S et al [32] reviewed the recent progress of transfer learning modelling and classified transfer learning into three categories based on the way of knowledge transfer: instance-based knowledge transfer [34], feature-based knowledge transfer [35] and parameter-based knowledge transfer [36].

Transfer learning modelling is generally conducted around three central dogmas: (1) how to define the relatedness between domains; (2) what to transfer; (3) how to transfer. In our work, we explicitly define the relatedness between protein sub-families and super-families by protein sequence evolution, i.e. protein homolog. Evolutionally closely-related proteins share similar subcellular localization patterns with high probability. Correspondingly, what to transfer is naturally the homolog $G O$ term. Such a way of transfer learning modelling is computationally simple and biologically interpretable. In order to reduce the risk of negative transfer, GO-TLM [25] and $M K-T L M$ [26] proposed a nonparametric multiple kernel learning method to measure the contribution of $G O$ three aspects, target $G O$ information and homolog $G O$ information to the model performance. In this paper, we redefine confusion matrix, so that the $G O$ kernel weights can be derived by cross validation for multi-label learning scenario.

\section{GO feature construction}

All the proteins are represented with both the target $G O$ terms and the homolog $G O$ terms, which are extracted from $G O A$ database [12] (77 Release, as of 30 November, 2009), and the homologs are extracted from SwissProt 57.3 database [13] using PSI-Blast [37]. Assume there are $u G O$ terms $x_{i}(i=1,2, \ldots, u)$, then protein $X$ can be represented as follows:

$$
X=x_{1} x_{2} \ldots x_{u}
$$

If $G O$ term $x_{i}$ is assigned to the protein $x$ in $G O A$ database, then $x_{i}=1$; Otherwise, $x_{i}=0$. To expressly estimate the individual contribution of the three $G O$ aspects, GO-TLM [25] decomposed the feature vector (1) into the following three binary feature vectors:

$$
\begin{aligned}
X_{P} & =\left(x_{P, 1}, x_{P, 2}, \ldots, x_{P, l}\right) ; X_{F}=\left(x_{F, 1}, x_{F, 2}, \ldots, x_{F, m}\right) ; X_{C} \\
& =\left(x_{C, 1}, x_{C, 2}, \ldots, x_{C, n}\right)
\end{aligned}
$$

However, GO-TLM aggregated the target $G O$ information and the homolog $G O$ information into one single feature vector, such that the two kinds of $G O$ information are treated equally. We know that such a way of feature construction is not rational because divergent homolog $G O$ information carries much noise. Figure 1 shows the difference of subcellular localization patterns between target human protein (P61221 thru. Q91203) and its homolog protein. The homologs are queried against SwissProt 57.3 database [13] with default Blast options (E-value: 10; substitution matrix: BLOSUM62). E-value is relaxed to 10 to obtain remote homologs for those proteins that have no significant homologs. For a target protein, we may encounter three cases for the selected homologs: 


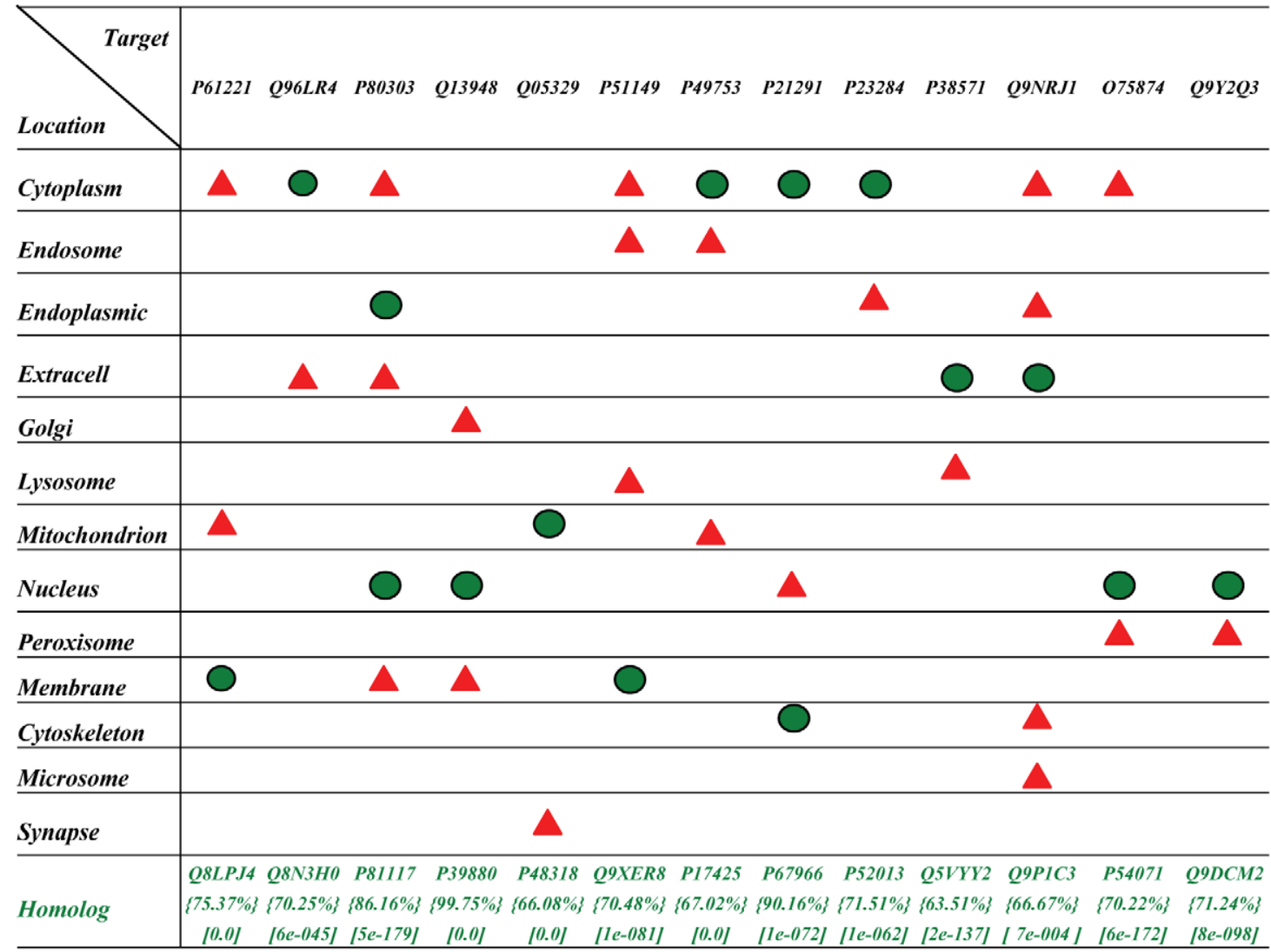

\section{Illustrations:}

$\Lambda$ : denotes the cellular compartments of target protein;

denotes the cellular compartments of homolog;

Q8LPJ4 \{75.37\% 3 [0.0]: the protein accession in green and italic denotes homolog; the curly braced number denotes sequence identity; the square bracketed number denotes PSI-Blast E-value;

Figure 1. Illustration of divergent homolog in terms of subcellular localization.

doi:10.1371/journal.pone.0037716.g001

(1) all homologs are significant homologs; (2) one part of homologs is significant homolog and the other part of homologs is remote homolog; (3) all homologs are remote homologs. Some remote homologs are convergent to the target protein in terms of protein subcellular localization (e.g. remote homologs 075881, 002766, Q63688, P22680, Q16850, O88962 and Q64505 to target protein P21291), thus we should exploit the useful information from remote homologs; meanwhile, some remote homologs are divergent to the target protein, thus we should prevent negative knowledge transfer from the remote homolog. As compared to remote homolog, significant homolog is more likely to be convergent in terms of protein subcellular localization, but in some case, significant homolog is also likely to be divergent. Figure 1 lists one divergent homolog for each target protein. The illustrated divergent homolog has the highest sequence identity and PSI-Blast E-value among the target protein's divergent homologs. From Figure 1, we can see that the significant homologs reside in definitely distinct subcellular locations from the target protein, which implies that we should also depress noise from the significant homologs even though we encounter the above case (1). Similar to $M K-T L M$ [26], we also separate the target $G O$ information from its homolog $G O$ information for the convenience of noise control. Here, we use $T$ to denote the target protein and $H$ to denote its homolog, thus the target $G O$ feature vector is expressed as formula (3), and the homolog $G O$ terms are aggregated into one homolog feature vector as formula (4):

$$
\begin{aligned}
X_{P}^{T} & =\left(x_{P, 1}, x_{P, 2}, \ldots, x_{P, l}\right) ; X_{F}^{T}=\left(x_{F, 1}, x_{F, 2}, \ldots, x_{F, m}\right) ; X_{C}^{T} \\
& =\left(x_{C, 1}, x_{C, 2}, \ldots, x_{C, n}\right) \\
X_{P}^{H}(x) & =\left(x_{P, 1}, x_{P, 2}, \ldots, x_{P, l}\right) ; X_{F}^{H}(x) \\
& =\left(x_{F, 1}, x_{F, 2}, \ldots, x_{F, m}\right) ; X_{C}^{H}(x)=\left(x_{C, 1}, x_{C, 2}, \ldots, x_{C, n}\right)
\end{aligned}
$$

Thus, each protein is represented by six binary feature vectors: $X_{P}^{T}, X_{F}^{T}, X_{C}^{T} ; X_{P}^{H}, X_{F}^{H}, X_{C}^{H}$.

\section{Non-parametric multiple kernel learning}

The six binary $G O$ feature vectors $\left\{X_{P}^{T}, X_{F}^{T}, X_{C}^{T} ; X_{P}^{H}, X_{F}^{H}, X_{C}^{H}\right\}$ are used to derive six $G O$ kernels $\left\{K_{P}^{T}, K_{F}^{T}, K_{C}^{T} ; K_{P}^{H}, K_{F}^{H}, K_{C}^{H}\right\}$, and the $G O$ kernels are further combined in the way that $M K-T L M$ does [26]. In such a setting, higher homolog $G O$ kernel weight implies more positive knowledge transfer, and lower homolog $G O$ kernel weight can depress the potential noise by divergent homolog. Different to $M K-T L M, M L M K-T L M$ adapts confusion matrix to multi-label learning scenario based on the concept of locative protein $[4,5]$. For self-contained description and integrity, we give the full description of non-parametric kernel weight estimation in multi-label learning scenario as below, though some part of which 
Table 1. Optimal performance on 3681 human locative protein dataset.

\begin{tabular}{|c|c|c|c|c|c|c|c|c|c|c|}
\hline \multirow{2}{*}{ Subcellular location } & \multirow{2}{*}{ Size } & \multicolumn{3}{|c|}{$\begin{array}{l}\text { MLMK-TLM-I (optimistic) } \\
\qquad\left(H=1 ; \gamma=2^{-1} ; C=2^{8}\right)\end{array}$} & \multicolumn{3}{|c|}{$\begin{array}{l}\text { MLMK-TLM-II (moderate) } \\
\qquad\left(H=1 ; \gamma=2^{-1} ; C=2^{8}\right)\end{array}$} & \multicolumn{3}{|c|}{$\begin{array}{l}\text { MLMK-TLM-III (pessimistic) } \\
\qquad\left(H=1 ; \gamma=2^{-1} ; C=2^{8}\right)\end{array}$} \\
\hline & & $S P$ & $S E$ & MCC & $S P$ & $S E$ & MCC & $S P$ & $S E$ & MCC \\
\hline Centrosome & 77 & 0.9063 & 0.7532 & 0.8229 & 0.8772 & 0.6494 & 0.7504 & 0.8235 & 0.7273 & 0.7694 \\
\hline Cytoplasm & 817 & 0.7845 & 0.8556 & 0.7704 & 0.8061 & 0.8042 & 0.7552 & 0.7380 & 0.8446 & 0.7322 \\
\hline Cytoskeleton & 79 & 0.9123 & 0.6582 & 0.7710 & 0.7910 & 0.6709 & 0.7231 & 0.8333 & 0.6329 & 0.7212 \\
\hline Endosome & 24 & 0.9167 & $\underline{0.4583}$ & 0.6467 & 0.8000 & $\underline{0.5000}$ & 0.6306 & 0.9167 & $\underline{0.4583}$ & 0.6467 \\
\hline Endoplasmic reticulum & 229 & 0.9302 & 0.8734 & 0.8951 & 0.9151 & 0.8472 & 0.8730 & 0.8818 & 0.8472 & 0.8557 \\
\hline Extracell & 385 & 0.9525 & 0.8857 & 0.9096 & 0.9284 & 0.8753 & 0.8906 & 0.9413 & 0.8753 & 0.8977 \\
\hline Golgi apparatus & 161 & 0.9214 & 0.8012 & 0.8534 & 0.9161 & 0.8137 & 0.8576 & 0.8705 & 0.7516 & 0.8010 \\
\hline Lysosome & 77 & 1.0000 & 0.7143 & 0.8426 & 0.9828 & 0.7403 & 0.8504 & 0.9825 & 0.7273 & 0.8426 \\
\hline Microsome & 24 & 0.9500 & 0.7917 & 0.8665 & 0.8947 & 0.7083 & 0.7949 & 0.8947 & 0.7083 & 0.7949 \\
\hline Mitochondrion & 364 & 0.9620 & 0.9038 & 0.9255 & 0.9477 & 0.8956 & 0.9131 & 0.9339 & 0.8544 & 0.8825 \\
\hline Nucleus & 1021 & 0.8479 & 0.9334 & 0.8486 & 0.8156 & 0.9314 & 0.8238 & 0.8287 & 0.8815 & 0.8028 \\
\hline Peroxisome & 47 & 0.9750 & 0.8298 & 0.8983 & 0.9318 & 0.8723 & 0.9004 & 0.9762 & 0.8723 & 0.9219 \\
\hline Plasma membrane & 354 & 0.8746 & 0.8672 & 0.8576 & 0.8169 & 0.8446 & 0.8130 & 0.8680 & 0.8362 & 0.8370 \\
\hline Synapse & 22 & 1.0000 & $\underline{0.5455}$ & 0.7375 & 1.0000 & $\underline{0.5455}$ & 0.7375 & 1.0000 & $\underline{0.5000}$ & 0.7060 \\
\hline \multicolumn{2}{|l|}{ Overall Accuracy/MCC } & \multicolumn{3}{|c|}{$87.04 \% / 0.8606$} & \multicolumn{3}{|c|}{$85.22 \% / 0.8411$} & \multicolumn{3}{|c|}{ 83.97\%/0.8277 } \\
\hline
\end{tabular}

is identical to $M K-T L M[26]$. Similar to GO-TLM and $M K-T L M$, the final kernel is defined as the following linear combination of sub-kernels:

$$
\begin{gathered}
K_{M L M K-T L M}=\sum_{t \in\{T, H\}} \sum_{s \in\{P, F, C\}} w_{s}^{t} \times K_{s}^{t} \\
w_{u}^{v}=S E_{u}^{v} \times M C C_{u}^{v} / \sum_{t \in\{T, H\}} \sum_{s \in\{P, F, C\}} S E_{s}^{t} \times M C C_{s}^{t} \\
u \in\{P, F, C\}, v \in\{T, H\}
\end{gathered}
$$

Where $S E$ denotes recall rate or sensitivity and $M C C$ denotes Matthew's correlation coefficient. The kernel weights $w_{u}^{v} u \in\{P, F, C\}, v \in\{T, H\}$ are derived by cross validation. Given a training dataset, we divide the training set into $k$-fold disjoint parts. For each fold cross validation, one part is used as validation set and the other parts are merged as training set to train the combined-kernel SVM. Thus, we can derive a confusion matrix $M$ by evaluating the trained SVM against the test set. From the confusion matrix $M$, we can derive the kernel's $S E$ and $M C C$ measure as follows:

$$
\begin{aligned}
& p_{l}=M_{l, l}, q_{l}=\sum_{i=1, i \neq l}^{L} \sum_{j=1, j \neq l}^{L} M_{i, j}, r_{l}=\sum_{i=1, i \neq l}^{L} M_{i, l}, s_{l}=\sum_{j=1, j \neq l}^{L} M_{l, j} \\
& p=\sum_{l=1}^{L} p_{l}, q=\sum_{l=1}^{L} q_{l}, r=\sum_{l=1}^{L} r_{l}, s=\sum_{l=1}^{L} s_{l} \\
& S E=\sum_{l=1}^{L} M_{l, l} / \sum_{i=1}^{L} \sum_{j=1}^{L} M_{i, j} \\
& M C C=(p q-r s) / \sqrt{(p+r)(p+s)(q+r)(q+s)}
\end{aligned}
$$

Where, $M_{i, j}$ records the counts that class $i$ are classified to classj; superscript $L$ denotes subcellular locations; and all the other variables are intermediate variables that can be derived from the confusion matrix $M$.

In single-label learning scenario, $M_{i, j}(i \neq j)$ records the counts that class $i$ are misclassified to class $j$, which is not applicable to multi-label learning scenario. Let's borrow the notion of locative protein $[4,5]$ to describe the multi-label confusion matrix. Assume that a protein $p$ is located at two subcellular locations $\left\{C_{1}, C_{2}\right\}$, i.e., $p \in S_{C_{1}} \wedge p \in S_{C_{2}}\left(S_{C_{1}}, S_{C_{2}}\right.$ denote the set of proteins that reside in $C_{1}, C_{2}$, respectively), the notion of locative protein means that protein $p$ can be viewed as two different proteins $p_{1}, p_{2}\left(p_{1} \in S_{C_{1}} \wedge p_{2} \in S_{C_{2}}\right)$. Now take $p_{1}$ protein as test protein and the trained $S V M$ labels $p_{1}$ as follows:

$$
C=\operatorname{maxarg}_{l}\left(f\left(p_{1}, l\right) \mid l=1, \ldots, L\right)
$$

Where, $f\left(p_{1}, l\right)$ denotes the probability that protein $p_{1}$ is assigned the label $l$ (see Section 4 of Methods for how to derive probability outputs). Thus, the multi-label confusion matrix can be defined as follows:

$$
\begin{cases}M\left(C_{1}, C_{1}\right)=M\left(C_{1}, C_{1}\right)+1 & C \in\left\{C_{1}, C_{2}\right\} \\ M\left(C_{1}, C\right)=M\left(C_{1}, C\right)+1 & C \notin\left\{C_{1}, C_{2}\right\}\end{cases}
$$

Formula (9) shows that only if the predicted label of locative protein $p_{1}$ hits its true label $C_{1}$ or $C_{2}$, the prediction is deemed as correct; otherwise, the prediction would be deemed as incorrect.

As regards with kernel $K_{s}^{t} \quad s \in\{P, F, C\}, t \in\{T, H\}$, Gaussian kernel is used here:

$$
K_{s}^{t}(x, y)=\exp \left(\gamma|x-y|^{2}\right) \quad s \in\{P, F, C\}, t \in\{T, H\}
$$




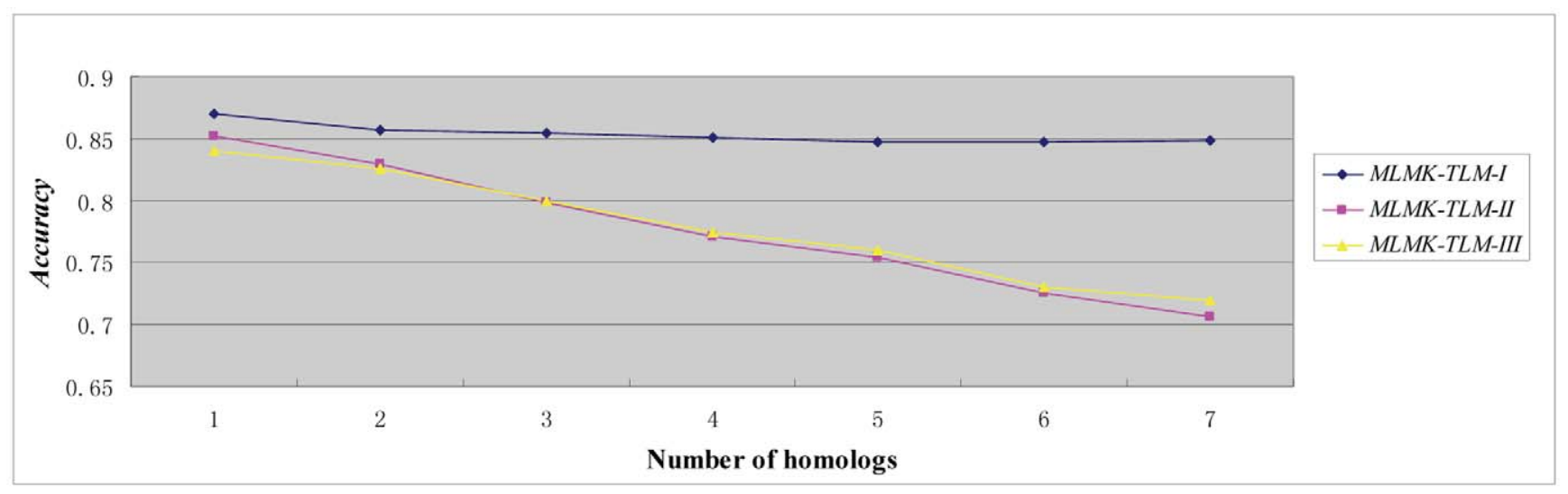

Figure 2. Performance on 3681 human protein dataset with varying homologs. doi:10.1371/journal.pone.0037716.g002

\section{Multi-label learning}

In our work, we extend $M K-T L M$ [26] to multi-learning scenario based on one-against-all multi-class learning and binary SVM probability outputs [38]. Probability outputs tell us the confidence level that a query protein belong to each subcellular location, thus more intuitive and reasonable than ensemble voting $[4,5,39]$ and label transfer of $k \mathcal{N N}$ nearest neighbour protein $[27,28,30]$.

Assuming there are $K$ subcellular locations, for each subcellular location $k$, we view the proteins that belong to $k$ as positive examples and the proteins that belong to other subcellular locations except $k$ as negative examples, based on which to train one binary SVM. Thus, we have $K$ trained binary $S V M$ s. If each binary $S V M$ outputs $\{-1,+1\}$ labels, multiple $\{+1\}$ outputs can be viewed as multiple protein subcellular locations [40]. Because the $\{-1,+1\}$ labels can not tell us the confidence level that a query protein belongs to each subcellular location, we don't adopt the method. If each binary SVM yields probability output, we can choose the label with the highest probability as the protein subcellular location, which is s-called oneagainst-all multi-class learning $[38,41]$; if we set some probability threshold, the labels with probability over the threshold can be viewed as multiple protein subcellular locations, thus intuitively applicable to multi-label learning scenario. Platt J [41] proposed a method to adapt binary $S V M\{-1,+1\}$ labels to posterior class probability as defined below:

$$
h_{y}(x)=p(y \mid x)=1 /\left(1+e^{A f(x)+B}\right)
$$

Where the coefficient $A$ and $B$ can be derived from data by cross validation, and $f(x)$ is uncalibrated decision value of binary $S V M$.

Actually, the one-against-all multi-class $S V M$ with probability output has been implemented into the LIBSVM tool (http://www. csie.ntu.edu.tw/ cjlin/libsvm/), which can be easily used for multi-label learning. Only if we set LIBSVM prediction option "-b 1 " (LIBSVM command option -b 1 means probability rather than $\{-1,+1\}$ output), we can obtain the probability vector that a query protein is predicted to each subcellular location. By setting optimal probability threshold, we can determine the optimal multiple labelling for the query protein based on the predicted probability vector.

\section{Model evaluation and model selection}

The existing GO-based models only reported the optimistic performance by evaluating the proposed model against information-rich $(G O, P P I$, image) test proteins, and seldom reported the performance for novel proteins $[4,5,14,15,16,17,18,19,20,21,22$, $23,24,25,27,28,29,30]$. Apparently, the optimistic performance is not enough to be a comprehensive survey of the model's true predictive ability, especially for novel protein prediction. $M K-T L M$ [26] attempted to conduct a comprehensive survey of the model performance in optimistic, moderate and pessimistic cases, and demonstrated good performance for novel proteins and those proteins that belong to the protein family we know little about. In this paper, the proposed $M L M K$-TLM inherits all $M K-T L M$ s advantages. The Optimistic case means the training set and the test set both abound in $G O$ information; the Moderate case means that the test set contains no $G O$ information at all, which can be simulated by removing the test kernels $\left\{K_{P}^{T}, K_{F}^{T}, K_{C}^{T}\right\}$; the Pessimistic case means that both the training set and the test set contains no $G O$ information at all where the target $G O$ information is removed from both the training set and the test set, which can be simulated by removing the training kernels $\left\{K_{P}^{T}, K_{F}^{T}, K_{C}^{T}\right\}$ and test kernels $\left\{K_{P}^{T}, K_{F}^{T}, K_{C}^{T}\right\}$.

The performance evaluation under multi-label learning scenario seems more complicated as compared to single-label learning scenario. Because the model performance estimation involves both singlex protein (only one subcellular location) and multiplex protein (multiple subcellular locations), we should conduct two performance estimation experiments: one experiment is overall performance estimation on locative dataset, where multiplex protein is viewed as multiple singlex proteins as Hum-mPLoc 2.0 [4], VirusmPLoc [15], iLoc-Euk [27], iLoc-Virus [28] and Plant-mPLoc [30] did; the other experiment is multi-labelling estimation for multiplex proteins. The first experiment is similar to traditional supervised learning estimation except that multi-label confusion matrix is adopted instead (see formula 8 \& 9); in the second experiment, cross validation is conducted on multiplex proteins only and the singlex proteins are always treated as training data. Thus, the whole training set is composed of two parts: fixed part from the singlex proteins and the variable part from the multiplex proteins. In addition, the model performance estimation in the second experiment is much more complicated. To simplify the formulation, lets' first give several symbol annotations: (1) $L_{\text {true }}$ denotes the true label set of a multiplex protein $p$; (2) $L_{\text {predicted }}$ denotes the predicted label set of a multiplex protein $p$; (3) $P\{p \mid F\}$ denotes the protein set $P$ whose protein $p$ satisfies the condition $F$; (4) $\llbracket \llbracket$ denotes set cardinal; (5) minus symbol - denotes set difference; (6) $\wedge$ denotes logic $A N D$. Based on the symbols, we can formally define Label Hit Rate (LHR), Perfect Label Match Rate (PLMR) and Non-target Label Hit Rate (NT-LHR) as follows: 


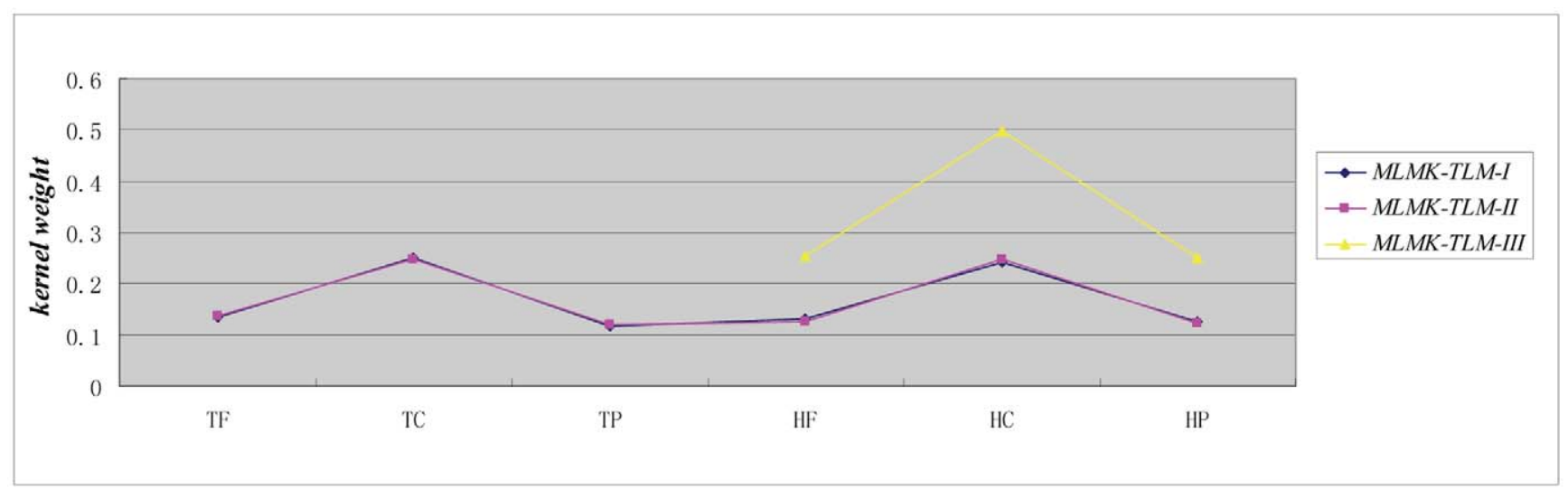

Figure 3. Kernel weight estimation on 3681 human locative protein dataset. doi:10.1371/journal.pone.0037716.g003

$$
\left\{\begin{array}{c}
L H R(n)=\llbracket P\left\{p \mid \llbracket L_{\text {true }} \cap L_{\text {predicted }} \rrbracket=n\right\} \rrbracket / \llbracket P\left\{p \mid \llbracket L_{\text {true }} \rrbracket=N\right\} \rrbracket \\
n=0,1 \ldots, N ; N=2,3,4 \\
\llbracket P\left\{p \mid\left(L_{\text {true }}-L_{\text {predicted }}=\varnothing\right) \wedge\right. \\
P L M R(N)=\left(L_{\text {predicted }}-L_{\text {true }}=\varnothing\right) \wedge \\
\left.\llbracket L_{\text {true }} \rrbracket=N\right\} \rrbracket
\end{array} \quad \begin{array}{c}
\llbracket P\left\{p \mid \llbracket L_{\text {true }} \rrbracket\right. \\
=N\} \rrbracket
\end{array}\right.
$$

where $\mathcal{N}$ denotes the number of subcellular locations a protein may reside in, with maximum value 4 here; $n$ denotes the number of correct HITs or wrong HITS, with maximum value $14-N$ here (we assume the total number of subcellular locations is 14). The multiplex proteins in Hum-mPLoc 2.0 [5] can be divided into 3 subsets that possesses 2, 3 and 4 labels (subcellular locations), respectively. We will report LHR, PLMR and NT-LHR on each subset. Take 2-label subset as example, the prediction may hit 0,1 or 2 true labels. Low 0 label hit rate and high 1- and/or 2-label hit rate imply good model performance. However, the prediction may also hit $1 \sim 12$ non-target labels (excluding 2 true labels from total 14 subcellular locations). High NT-LHR implies high misleading tendency, which should be as low as possible. The existing multi-label learning model for protein subcellular localization $[4,5,15,19,27,28,29,30]$ seldom reported $\mathcal{N}$ $L H R$. If the prediction hits the true labels and yields no other misleading labels, we call the case perfect label match; otherwise, we call the case non-perfect label match. High Perfect Label Match Rate (PLMR) implies good predictive ability and low misleading tendency.
$M L M K-T L M$ is a relatively complex model that requires timeconsuming computation for model comparison and model selection. Apart from SVM regularization parameter $C$ and kernel parameter $\gamma, M L M K-T L M$ introduces a hyper-parameter $H$ that denotes the number of homologs for knowledge transfer. Assume there are $\mathcal{N}$ proteins in the dataset and the hyper-parameter sets are $C=\left\{2^{3}, 2^{4}, 2^{5}, 2^{6}, 2^{7}, 2^{8}, 2^{9}, 2^{10}, 2^{11}\right\} ; \gamma=\left\{2^{-3}, 2^{-2}, 2^{-1}\right\} ;=\{1$, 2,3,4,5,6,7,8,9,10\}, MLMK-TLM has to fix one hyper-parameter to optimize the other hyper-parameters, and in each iteration has to compute kernel matrices, thus the computational complexity is $\llbracket K \rrbracket \times \llbracket C \rrbracket \times \llbracket \gamma \rrbracket \times \llbracket H \rrbracket \times \mathrm{O}\left(N^{2}\right)$, where $\llbracket \bullet \rrbracket$ denotes set cardinal, $\llbracket K \rrbracket$ denotes the number of kernel matrices and $\mathrm{O}\left(N^{2}\right)$ denotes the computational complexity for kernel computing. For large-scale human protein dataset Hum-mPLoc 2.0 [5], the model selection is rather time-consuming. Hence, we adopt 5 -fold cross validation instead of leave-one-out cross validation (LOOCV) (Jackknife) as GO$T L M$ [25] and $M K-T L M$ [26] did. For multi-labelling estimation, the multiplex proteins are divided into 5 nearly-even parts, one part as test set, and the other parts are merged with the singlex proteins into training set, thus iterates for 5 times until all the multiplex proteins participate in the performance estimation process (see Section 6 of Results).

For performance estimation on locative proteins, we adopt the performance measures: Sensitivity $(S E)$, Specificity $(S P)$, Matthew's correlation coefficient $(M C C)$, Overall $M C C$, and Overall Accuracy. For multi-labelling estimation, we adopt LHR, PLMR and NT-LHR.

\section{Results}

\section{Dataset}

Shen $\mathrm{HB}$ et al [5] constructed a large-scale human protein dataset. The dataset covers 14 subcellular locations and contains

\begin{tabular}{|c|c|c|c|c|c|c|c|c|c|c|c|}
\hline \multirow[t]{2}{*}{$\begin{array}{l}\text { Multiplex } \\
\text { Locations }\end{array}$} & \multirow[t]{2}{*}{ Size } & \multicolumn{5}{|c|}{ Label Hit Rate } & \multirow{2}{*}{ PLMR } & \multicolumn{4}{|c|}{ Non-target Label Hit Rate } \\
\hline & & 0 & 1 & 2 & 3 & 4 & & 1 & 2 & 3 & 4 \\
\hline 2 & 480 & $3.33 \%$ & $38.12 \%$ & $58.54 \%$ & 0 & 0 & $42.92 \%$ & $18.96 \%$ & $8.54 \%$ & $\underline{2.92 \%}$ & $\underline{0.42 \%}$ \\
\hline 3 & 43 & $2.33 \%$ & $25.58 \%$ & $44.19 \%$ & $27.91 \%$ & 0 & $13.95 \%$ & $\underline{25.58 \%}$ & $\underline{16.28 \%}$ & $\underline{2.33 \%}$ & 0 \\
\hline 4 & 3 & 0 & $33.33 \%$ & 0 & $33.33 \%$ & $33.33 \%$ & $33.33 \%$ & 0 & 0 & $33.33 \%$ & 0 \\
\hline
\end{tabular}
3106 distinct human proteins, where 2580 proteins belong to one

Table 2. Multi-labelling evaluation for optimistic case.

doi:10.1371/journal.pone.0037716.t002 
Table 3. Multi-labelling evaluation for moderate case.

\begin{tabular}{|c|c|c|c|c|c|c|c|c|c|c|c|}
\hline \multirow[t]{2}{*}{$\begin{array}{l}\text { Multiplex } \\
\text { Locations }\end{array}$} & \multirow[t]{2}{*}{ Size } & \multicolumn{5}{|c|}{ Label Hit Rate } & \multirow[t]{2}{*}{ PLMR } & \multicolumn{4}{|c|}{ Non-target Label Hit Rate } \\
\hline & & 0 & 1 & 2 & 3 & 4 & & 1 & 2 & 3 & 4 \\
\hline 2 & 480 & $4.37 \%$ & $38.75 \%$ & $56.87 \%$ & 0 & 0 & $38.75 \%$ & $\underline{22.29 \%}$ & $9.38 \%$ & $3.96 \%$ & $0.63 \%$ \\
\hline 3 & 43 & 0 & $25.58 \%$ & $48.84 \%$ & $25.58 \%$ & 0 & $9.30 \%$ & $\underline{20.93 \%}$ & $\underline{25.58 \%}$ & $\underline{2.33 \%}$ & 0 \\
\hline 4 & 3 & 0 & $33.33 \%$ & $33.33 \%$ & $33.33 \%$ & 0 & 0 & $33.33 \%$ & $33.33 \%$ & 0 & 0 \\
\hline
\end{tabular}

subcellular location, 480 to two locations, 43 to three locations, and 3 to four locations. The protein with multiple subcellular locations should be treated as one training example of each subcellular location it belongs to, thus the same protein should be viewed as different protein within different subcellular location, referred to as locative protein in the literatures $[4,5,15,19,27,28,29,30]$. Thus, there are 3681 locative proteins in the dataset [5]. The dataset is a good benchmark for model performance comparison, because none of the proteins has $\geq 25 \%$ sequence identity to any other proteins in the same subcellular location. Accordingly, we choose Hum-mPLoc 2.0 [5] as the baseline models for performance comparison. Although the dataset [40] collected much more multiplex human proteins, we don't use it to evaluate the multi-labelling, because the sequence similarity reaches $80 \%$, so high as to yield performance overestimation.

\section{Model performance evaluation}

2.1 Optimistic case: both training set and test set abound in target $\boldsymbol{G} \boldsymbol{O}$ information. The optimistic case assumes that both the training set and the test set abound in target $G O$ information, that's, the training proteins and the test protein by themselves contain rich $G O$ information before incorporating the homolog $G O$ information. We call this case $M L M K-T L M-I$. As shown in $M L M K-T L M-I$ section of Table 1, MLMK-TLM achieves $87.04 \%$ accuracy and $0.8606 M C C$ on Hum-mPLoc 2.0 human protein data, significantly outperforming the baseline Hum-mPLoc $2.062 .7 \%$ [5]. Actually, Hum-mPLoc 2.0 aggregated the target protein's $G O$ information together with the homolog $G O$ information to train classifier, thus the overall accuracy $62.7 \%$ is the model's optimistic performance. The optimal hyper-parameter setting is $\left(H=1 ; \gamma=2^{-1} ; C=2^{8}\right)$, where $H=1$ means that only one homolog $G O$ information is transferred to the target protein. The high $M C C$ value (0.8606) implies that $M L M K-T L M$ achieves good predictive balance among the 14 human protein subcellular locations. We can see from $M L M K-T L M-I$ section of Table 1 that $M L M K-T L M$ achieves good model performance one most subcellular locations, even the small Peroxisome $(S P=0.9750$;
$S E=0.8298 ; \quad M C C=0.8983) \quad$ and Microsome $\quad(S P=0.9500$; $S E=0.7917 ; M C C=0.8665) . \quad M L M K-T L M$ relatively underperforms on the small subcellular locations: Endosome $(S P=0.9167$; $S E=0.4583 ; \quad M C C=0.6467) \quad$ and Synapse $\quad(S P=1.0000$; $S E=\overline{0.5455} ; M C C=0.7375)$. The poor performance may result from the sources: (1) small number of training proteins; (2) the target $G O$ information is not as rich as the other subcellular locations; (3) the homolog $G O$ information may be more divergent.

2.2 Moderate case: training set abounds in target $G O$ information while test set contains no target $G O$ information. The most common scenario we encounter may be that we have a plenty of well-annotated training proteins and need to label some novel proteins at hand. We call the scenario as moderate case, referred to as MLMK-TLM-II. Novel proteins generally have no $G O$ information at all. Most of the existing GO-based models except the work [26] ignored performance estimation in this case. Once the proposed models work in such a scenario, the performance may not be as optimistic as reported. Therefore, experiments should be expressly designed for the moderate case to test $M L M K-T L M$ 's applicability to novel proteins.

The test procedure for moderate case seems more complicated than that for optimistic case, because the proteins in the test set have no target $G O$ information. Thus, the three target test kernels $K_{T_{c}}^{\text {Test }}=\exp \left(\gamma\left|X_{T_{c}}^{\text {Test }}(x)-X_{T_{c}}^{\text {Train }}\right|^{2}\right), c \in\{P, F, C\}$ can not be derived, because $X_{T_{c}}^{\text {Test }}(x)$ is null (superscript Test denotes test set and Train denotes training set). For the reason, we substitute the homolog $G O$ feature vector of test protein for its target $G O$ feature vector to calculate the test kernel as follows:

$$
\begin{aligned}
K_{T_{c}}^{\text {Test }} & =\exp \left(\gamma\left|X_{H_{c}}^{\text {Test }}(x)-X_{T_{c}}^{\text {Train }}\right|^{2}\right) ; K_{H_{c}}^{\text {Test }} \\
& =\exp \left(\gamma\left|X_{H_{c}}^{\text {Test }}(x)-X_{H_{c}}^{\text {Train }}\right|^{2}\right), c \in\{P, F, C\}
\end{aligned}
$$

As shown in MLMK-TLM-II section of Table 1, MLMK-TLM achieves $85.22 \%$ accuracy and $0.8411 M C C$ on the benchmark data, still significantly outperforming the baseline Hum-mPLoc 2.0 $62.7 \%$ [5] and nearly $2 \%$ lower than the optimistic case $(87.04 \%$

\begin{tabular}{|c|c|c|c|c|c|c|c|c|c|c|c|}
\hline & \multirow[t]{2}{*}{$\begin{array}{l}\text { Multiplex } \\
\text { Locations }\end{array}$} & \multirow{2}{*}{$\begin{array}{l}\text { Size } \\
0\end{array}$} & \multicolumn{4}{|c|}{ Label Hit Rate } & \multirow[t]{2}{*}{$P L M R$} & \multicolumn{4}{|c|}{ Non-target Label Hit Rate } \\
\hline & & & 1 & 2 & 3 & 4 & & 1 & 2 & 3 & 4 \\
\hline 2 & 480 & $3.75 \%$ & $38.12 \%$ & $58.13 \%$ & 0 & 0 & $34.58 \%$ & $24.58 \%$ & $11.67 \%$ & $\underline{4.79 \%}$ & $2.50 \%$ \\
\hline 3 & 43 & 0 & $25.58 \%$ & $41.86 \%$ & $32.56 \%$ & 0 & $9.30 \%$ & $25.58 \%$ & $18.60 \%$ & $11.63 \%$ & 0 \\
\hline 4 & 3 & 0 & $33.33 \%$ & 0 & $66.67 \%$ & $33.33 \%$ & 0 & 0 & $33.33 \%$ & 0 & $33.33 \%$ \\
\hline
\end{tabular}

Table 4. Multi-labelling evaluation for pessimistic case. 
Table 5. Multi-labelling evaluation-perfect label match.

\begin{tabular}{|c|c|}
\hline & Protein Accessions \\
\hline $\begin{array}{l}\text { Optimistic case } \\
{[>=0.09]}\end{array}$ & 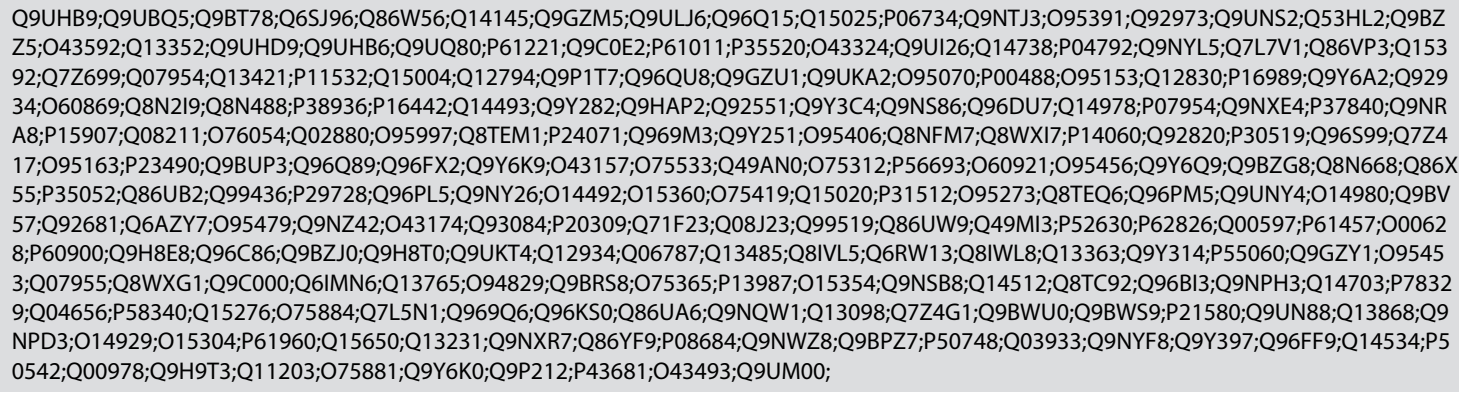 \\
\hline $\begin{array}{l}\text { Moderate case } \\
{[>=0.08]}\end{array}$ & 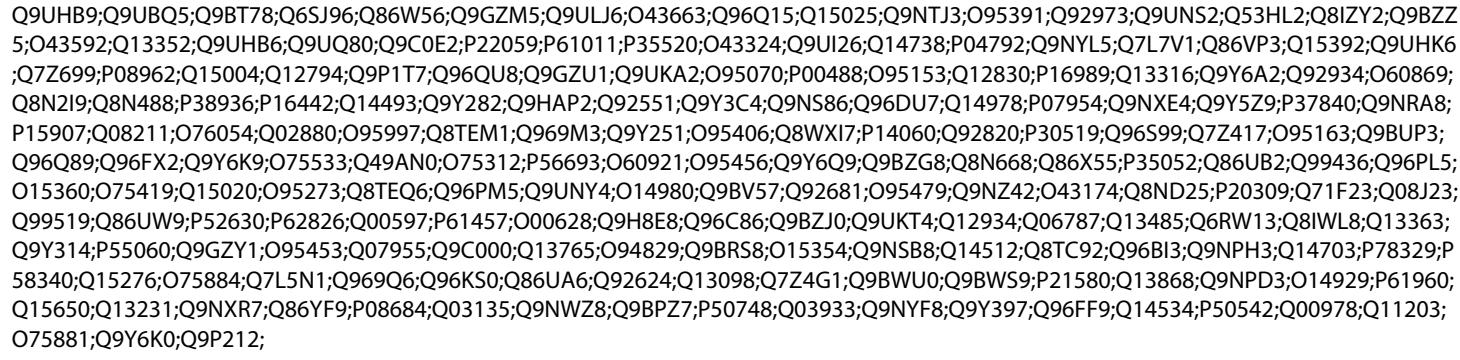 \\
\hline $\begin{array}{l}\text { Pessimistic case } \\
{[>=0.07]}\end{array}$ & $\begin{array}{l}\text { Q9UBQ5;Q9BT78;Q6SJ96;Q86W56;Q9UL6;O43663;Q96Q15;Q15025;P06734;Q9NTJ3;O95391;Q92973;Q9UNS2;Q53HL2;Q9BZZ5;O43592;Q13352; } \\
\text { Q9UHB6;Q9UQQ8;Q9COE2;P61011;P35520;O43324;Q9UI26;Q14738;P04792;Q9NY5;Q7L7V1;Q15392;Q9UHK6;Q7Z699;P11532;Q15004;Q12794; } \\
\text { Q9P1T7;Q96QU8;Q9UKA2;A5X5Y0;P00488;O95153;Q12830;P16989;Q9Y6A2;Q92934;O60869;Q8N488;P38936;P16442;Q14493;Q9HAP2;Q92551; } \\
\text { Q9Y3C4;Q9NS86;Q96DU7;Q14978;P07954;Q9NXE4;Q9Y5Z9;P37840;Q9NRA8;P15907;Q08211;O76054;Q02880;O95997;Q9UKL3;P24071;Q969M3; } \\
\text { Q9Y251;O95406;Q92820;P30519;Q96S99;Q7Z417;O95163;Q9BUP3;Q96Q89;Q96FX2;O75533;Q49AN0;P56693;O60921;O95456;Q9Y6Q9;Q9BZG8; } \\
\text { Q86X55;P35052;P58335;Q86UB2;Q99436;Q96PL5;O15360;O75419;Q15020;O95273;Q8TEQ6;Q96PM5;Q9UNY4;O14980;Q9BV57;Q92681;Q9NZ42; } \\
\text { O43174;P20309;Q71F23;Q08J23;Q86UW9;P52630;P62826;Q00597;P61457;Q9H8E8;Q96C86;Q9BZJ0;Q08378;Q9UKT4;Q06787;Q13485;Q8IWL8;Q } \\
\text { 13363;Q9Y314;P55060;O95453;Q07955;Q9C000;Q6IMN6;Q13765;O94829;Q9BRS8;Q9UKT7;P49721;Q14512;Q8TC92;Q96BI3;Q9NPH3;Q14703;P8 } \\
\text { 329;Q04656;P58340;O75884;Q7L5N1;Q969Q6;Q96KS0;Q86UA6;Q92624;Q13439;Q13098;Q7Z4G1;Q9BWU0;Q9BWS9;P21580;Q13868;Q9NPD3;O } \\
\text { 14929;P61960;Q13231;Q9NXR7;P08684;Q9NWZ8;P50748;Q03933;Q9NY78;Q9Y397;Q96F9;Q14534;Q00978;Q53QV2;Q9H9T3;Q11203;O75881; }\end{array}$ \\
\hline
\end{tabular}

doi:10.1371/journal.pone.0037716.t005

accuracy; $0.8606 \mathrm{MCC})$. Except for small performance decrease, $M L M K-T L M-I I$ demonstrates similar behaviour to MLMK-TLM-I (predictive balance and relative poor performance on Endosome \& Synapse, see the underscored $S E$ measures). The results show that $M L M K-T L M$ is convincingly applicable to novel protein prediction, and the performance decrease shows that it is necessary to expressly report the model performance in moderate case.

2.3 Pessimistic case: both training set and test set contain no target $\boldsymbol{G} \boldsymbol{O}$ information. In this section, we study an extreme case, called pessimistic case, where a protein subfamily or species is not $G O$-annotated at all, that's, we know nothing about the protein subfamily or species but the protein sequence information. The key point is whether the homolog $G O$ information is informative enough to train an effective prediction model for the protein subfamily or species we know little about. To validate the point, we assume that at least one $G O$-annotated homolog can be queried for the target protein, which is not restrictive with the rapid progress of $G O A$ database [12]. If experimental results support the idea, $M L M K-T L M$ will gain much wider application. Different from the optimistic case and the moderate case, the pessimistic test procedure contains only three homolog $G O$ kernels with target $G O$ kernels missing.

As shown in MLMK-TLM-III section of Table 1, MLMK-TLM achieves $83.97 \%$ accuracy and $0.8277 M C C$ on the benchmark data, significantly outperforming the baseline Hum-mPLoc 2.0 $62.7 \%$ [5], nearly $3 \%$ lower than the optimistic case $(87.04 \%$ accuracy; $0.8606 \mathrm{MCC}$ ) and nearly $1.5 \%$ lower than the moderate case $(85.22 \%$ accuracy; $0.8411 M C G)$. Similarly, MLMK-TLM-II demonstrates similar behaviour to MLMK-TLM-I \& MLMK-TLM$I$ (predictive balance and relative poor performance on Endosome \& Synapse, see the underscored $S E$ measures). The results show that $M L M K-T L M$ is applicable to novel protein prediction for the protein subfamily or species that we know little about.

\section{Optimal number of homologs}

Homolog is a good bridge for knowledge transfer between two evolutionarily- related protein subfamilies, super-families or species. However, biological evidences demonstrate that divergent homologs are subjected to different subcellular localization patterns from the target protein (see Figure 1), thus incorporating divergent homologs would leads to negative transfer and do harm to model performance. Thus, it is highly required to quantitatively study how much homolog $G O$ information should be transferred to the target protein. Most of the existing Homolog-GO-based models except the work [26] seldom conducted the quantitative analysis. Because the homolog space is generally quite huge, the model selection is unendurably long if the hyper-parameter $H$ is large, so we empirically define the homolog search space as 7 homologs with the most significant $E$-value.

As shown in Figure 2, the optimal number of homologs is 1 for optimistic case (MLMK-TLM-I), moderate case (MLMK-TLM-II), and pessimistic case (MLMK-TLM-III). The model performance slightly decreases for the optimistic case (MLMK-TLM-I) with the incorporation of more homologs, while the model performance decreases 
Table 6. Multi-labelling evaluation-non-perfect label match.

\begin{tabular}{|c|c|c|c|}
\hline & & True Subcellular Locations & Predicted Subcellular Locations \\
\hline \multirow{8}{*}{$\begin{array}{l}\text { Optimistic Case } \\
{[>=0.09]}\end{array}$} & 043663 & Cytoplasm; Nucleus & Nucleus[0.471]; Cytoplasm[0.365]; Cytoskeleton[0.136] \\
\hline & Q92797 & Cytoskeleton; Nucleus & Nucleus[0.470]; Cytoplasm[0.257]; Plasma membrane[0.129] \\
\hline & Q92597 & Cytoplasm; Nucleus; Plasma membrane & Nucleus[0.588]; Cytoplasm[0.333] \\
\hline & P30533 & Cytoplasm; Endoplasmic reticulum & $\begin{array}{l}\text { Endoplasmic reticulum[0.237]; Cytoplasm[0.229] } \\
\text { Nucleus[0.115]; Plasma membrane[0.181] }\end{array}$ \\
\hline & Q9HD36 & Mitochondrion; Nucleus & Nucleus[0.562];Mitochondrion[0.160];Cytoplasm[0.156] \\
\hline & P22059 & Cytoplasm; Golgi apparatus & Golgi apparatus[0.837] \\
\hline & Q86YR5 & Endoplasmic reticulum; Golgi apparatus & $\begin{array}{l}\text { Golgi apparatus[0.454]; Endoplasmic reticulum[0.316]; } \\
\text { Cytoplasm[0.091] }\end{array}$ \\
\hline & P41222 & Endoplasmic reticulum; Golgi apparatus; Nucleus & $\begin{array}{l}\text { Endoplasmic reticulum[0.237]; Nucleus[0.207] } \\
\text { Extracell[0.146]; Cytoplasm[0.143]; Golgi apparatus[0.107]; }\end{array}$ \\
\hline \multirow{8}{*}{$\begin{array}{l}\text { Moderate Case } \\
{[>=0.08]}\end{array}$} & P15941 & Cytoplasm; Nucleus & Plasma membrane[0.467]; Nucleus[0.178]; Cytoplasm[0.145] \\
\hline & Q14145 & Cytoplasm; Nucleus & Cytoplasm[0.520]; Nucleus[0.292]; Endoplasmic reticulum[0.089] \\
\hline & Q86YR5 & Endoplasmic reticulum; Golgi apparatus & $\begin{array}{l}\text { Golgi apparatus[0.371]; Endoplasmic reticulum[0.272]; } \\
\text { Cytoplasm[0.129]; Plasma membrane[0.124] }\end{array}$ \\
\hline & P41222 & $\begin{array}{l}\text { Endoplasmic reticulum;Golgi apparatus } \\
\text { Nucleus }\end{array}$ & $\begin{array}{l}\text { Nucleus[0.257];Endoplasmic reticulum[0.214]; Cytoplasm[0.166]; } \\
\text { Extracell[0.103]; Golgi apparatus[0.098] }\end{array}$ \\
\hline & Q96JC1 & Cytoplasm; Lysosome & Lysosome[0.676]; Endosome[0.208] \\
\hline & Q96EY5 & Cytoplasm; Nucleus & $\begin{array}{l}\text { Nucleus[0.223]; Endosome[0.202]; Golgi apparatus[0.121] } \\
\text { Cytoskeleton[0.092] }\end{array}$ \\
\hline & Q86WA9 & $\begin{array}{l}\text { Endoplasmic reticulum;Golgi apparatus } \\
\text { Plasma membrane }\end{array}$ & $\begin{array}{l}\text { Lysosome[0.642]; Endoplasmic reticulum[0.136] } \\
\text { Plasma membrane[0.101] }\end{array}$ \\
\hline & Q9NWZ5 & Cytoplasm; Nucleus & Endoplasmic reticulum[0.459]; nucleus[0.320]; Cytoplasm[0.133] \\
\hline \multirow{8}{*}{$\begin{array}{l}\text { Pessimistic Case } \\
{[>=0.07]}\end{array}$} & Q9UHD9 & Cytoplasm; Nucleus & Endoplasmic reticulum[0.315]; Nucleus[0.284]; Cytoplasm[0.202] \\
\hline & P30533 & Cytoplasm; Endoplasmic reticulum & $\begin{array}{l}\text { Endoplasmic reticulum[0.250]; Cytoplasm[0.237] } \\
\text { Nucleus[0.151]; Plasma membrane[0.139] }\end{array}$ \\
\hline & Q86YR5 & Endoplasmic reticulum;Golgi apparatus & $\begin{array}{l}\text { Golgi apparatus[0.421]; Endoplasmic reticulum[0.202] } \\
\text { Cytoplasm[0.136]; Plasma membrane[0.106] }\end{array}$ \\
\hline & Q9NX74 & Cytoplasm; Endoplasmic reticulum & $\begin{array}{l}\text { Endoplasmic reticulum[0.273]; Cytoplasm[0.271] } \\
\text { Mitochondrion[0.247] }\end{array}$ \\
\hline & P41222 & $\begin{array}{l}\text { Endoplasmic reticulum;Golgi apparatus } \\
\text { Nucleus }\end{array}$ & $\begin{array}{l}\text { Nucleus[0.224]; Endoplasmic reticulum[0.197]; } \\
\text { Cytoplasm[0.184]; Extracell[0.126];Golgi apparatus[0.077] }\end{array}$ \\
\hline & Q96EY5 & Cytoplasm; Nucleus & $\begin{array}{l}\text { Nucleus[0.246]; Endosome[0.154]; Cytoskeleton[0.095]; Golgi } \\
\text { apparatus[0.119];Plasma membrane[0.094]; Endoplasmic } \\
\text { reticulum[0.075] }\end{array}$ \\
\hline & P42858 & Cytoplasm; Nucleus & $\begin{array}{l}\text { Nucleus[0.285]; Cytoplasm[0.216]; Endoplasmic reticulum[0.071] } \\
\text { Golgi apparatus[0.074]; Plasma membrane[0.096] }\end{array}$ \\
\hline & Q9Y613 & Cytoplasm; Cytoskeleton & Cytoskeleton[0.530]; Cytoplasm[0.146]; Nucleus[0.111] \\
\hline
\end{tabular}

Illustrations:

[1] True Subcellular Locations : denotes the labels from Hum-mPLoc 2.0 dataset (GOA database version 70.0 released March 10 2008);

[2] Cytoskeleton[0.136]:the label NOT included in the original Hum-mPLoc 2.0 dataset, but validated TRUE by the latest Swiss-Prot database (http://www.uniprot.org/uniprot/ UniProt release 2011_11 Nov 16, 2011), where [0.136] denotes the probability that the protein is assigned to the label Cytoskeleton;

[3] Nucleus[0.115]: Non-target Label Hit (wrong prediction), NOT validated by the latest Swiss-Prot database (http://www.uniprot.org/uniprot/ UniProt release 2011_11 Nov $16,2011)$, where [0.115] denotes the probability that the protein is assigned to the label Nucleus;

doi:10.1371/journal.pone.0037716.t006

sharply for the moderate (MLMK-TLM-II) \& pessimistic case $(M L M K-T L M-I I I)$. When the number of homologs reaches 7 , the accuracy sharply drops about $15 \%$ for moderate \& pessimistic case. We can see that divergent homologs adversely contribute little to the optimistic case, partly because the target protein's own $G O$ information can counteract the unfavourable impact of the divergent homolog $G O$ information. For the moderate \& pessimistic case, the unfavourable divergent homolog $G O$ information greatly deteriorates the model performance. From the results, we can safely conclude that it is highly necessary to quantitatively study how much homolog $G O$ information should be transferred to the target protein.

It is worthy noting that the pessimistic case contains no target $G O$ information but slightly outperforms the moderate case beyond our expectation (except at the first \& second points of the curve in Figure 2). The reason may be that the substitution of the homolog $G O$ feature vector for the target $G O$ feature vector results in the slight performance deterioration (see Formula 13). 


\section{Kernel weight distribution}

The GO kernel weights are evaluated using 3-fold cross validation as described in Section 3 of Methods, rather than 5fold cross validation as GO-TLM [25] conducted, because the additional hyper-parameter $H$ makes the model selection more time-consuming. Actually, to evaluate the model performance, we conduct two-level cross validation: the outer 5 -fold cross validation uses the whole dataset to evaluate performance, and the inner 3fold cross validation uses the training set from the outer cross validation to estimate the kernel weights. Similar to GO-TLM [25] and $M K-T L M$ [26], the kernel weight distributions yielded from the outer 5 -fold cross validation is quite similar, so we choose one typical kernel weight distribution to illustrate the $G O$ kernels' contribution to the model performance.

As shown in Figure 3, the $x$ axis denotes the six $G O$ kernels, where $T$ denotes target, $H$ denotes homolog, $F, C$ and $P$ denote the three aspects of gene ontology (molecular function, cellular compartment and biological process), respectively. We can see that both the optimistic case and the moderate case have similar kernel weight distributions on the benchmark dataset, while the pessimistic case is similar to the homolog $G O$ kernel weight distribution of the optimistic case and the moderate case (see the latter part of curve in Figure 3) (the pessimistic case contains only three homology $G O$ kernels in that the target protein's $G O$ information is missing). No matter the target $G O$ kernels or the homolog $G O$ kernels, $C$ (cellular component) demonstrates much higher kernel weight. For optimistic case and moderate case, both the target $G O$ kernels and the homolog $G O$ kernels make equivalent contribution to the model performance (compare the former half part and the latter half part of the curve in Figure 3). From the results, we can conclude that the homolog knowledge transfer is instrumental to novel target protein research.

\section{Multi-labelling estimation}

As stated in Section 4 of Methods, MLMK-TLM can yield the probability outputs from Formula 11 . We can assign to the test protein the subcellular locations whose predicted probability is greater than the optimal probability threshold. The threshold setting should achieve rational balance between higher LHR (Label Hit Rate) \& PLMR (Perfect Label Match Rate) and lower NT-LHR (Non-target Label Hit Rate) defined by Formula 12 in Section 5 of Methods. Generally, higher LHR \& PLMR also implies higher NT$L H R$. In the work, the optimal probability threshold is selected from $\{0.06,0.07,0.08,0.09,0.10,0.15,0.2\}$. Besides LHR, PLMR and NT-LHR (Table 2 thru. Table 4), we also list some proteins of perfect label match (Table 5) and non-perfect label match (Table 6) to demonstrate $M L M K-T L M$ 's multi-labelling ability. Interestingly, some non-target label hits in terms of the original Hum-mPLoc 2.0 dataset (GOA database version 70.0 released March 10 2008) are validated as TRUE LABEL (Correct Prediction) by the latest Swiss-Prot database (UniProt release 2011_11 Nov 16, 2011, http://www. uniprot.org/) (see Table 6).

As shown in Table 2 thru. Table 4, MLMK-TLM achieves $58.54 \%, 27.19 \%$ and 0 LHR (called complete label hit rate CLHR, in bold font) for 2, 3 and 4 multiple subcellular locations (optimistic case), respectively (see Table 2); $56.87 \%, 25.58 \% \%$ and $0 \mathrm{LHR}$ (CLHR, in bold font) for moderate case (see Table 3); and $58.13 \%$, $32.56 \%$ and $33.33 \%$ LHR (CLHR, in bold font) for pessimistic case (see Table 4). The results seem much more promising than $24.3 \%$ for 2-label hit rate, $3.6 \%$ for 3 -label hit rate and $6.7 \%$ for 4 -label hit rate, reported in the work [40]. The complete label hit rate (CLHR) for pessimistic case seems better than the optimistic \& moderate case, because of the probability thresholds: 0.09 for optimistic case, 0.08 for moderate case and 0.07 for pessimistic case. Relax probability threshold would yields higher Label Hit Rate $(L H R)$, but would yields higher Non-target Label Hit Rate (NT-LHR) at the same time. From Table 2 to Table 4, we can see that the pessimistic case shows higher NT-LHR than the optimistic E moderate case. The complete label hit means that all the true labels are correctly hit by the prediction, but it can not measure the model's misleading tendency, because the prediction is still likely to hit non-target labels. Perfect Label Match Rate $(P L M R)$ is the perfect measure that demonstrates the model's multi-labelling ability with zero misleading tendency. As shown thru. Table 2 to Table 4, we can see from PLMR measure that the optimistic case is the best $(42.92 \%, 13.95 \%, 33.33 \%)$, the moderate case the second $(38.75 \%, 9.30 \%, 0)$ and the pessimistic case the third $(34.58 \%, 9.30 \%, 0)$. We can see that even MLMK-TLM's Perfect Label Match Rate is much better than the Partial Label Match Rate that was reported in the work [40]. Table 5 lists all the proteins of perfect label match in optimistic, moderate and pessimistic case, and the detailed probability outputs for the perfect label match proteins see Supporting Information (File S1 for optimistic case, File S2 for moderate case and File S3 for pessimistic case).

To further demonstrate $M L M K-T L M$ 's multi-labelling ability, we list some proteins of non-perfect label match in Table 6 to show how the prediction varies from the true labels. Table 6 takes only 8 proteins for example and the full list of non-perfect label match proteins see Supporting Information (File N S1 for optimistic case, File N S2 for moderate case and File N S3 for pessimistic case). Take protein 043663 in the optimistic case as an example, 043663 is labelled Cytoplasm \& Nucleus in the original Hum-mPLoc 2.0 dataset [5] (GOA database version 70.0 released March 10 2008), and the prediction not only hits the two true labels but also hit a non-target label Cytoskeleton with probability 0.136. From the latest Swiss-Prot database (UniProt release 2011_11 Nov 16, 2011, http://www. uniprot.org/), we can see that Cytoskeleton is truly assigned to protein 043663 . The non-target labels validated as TRUE Label are underlined in Table 6 . We can see that there are many underlined TRUE Labels in Table 6 for optimistic, moderate and pessimistic case. For example, Cytoplasm [0.166] \& Extracell [0.103] for P41222 in the moderate case; Endoplasmic reticulum [0.071], Golgi apparatus [0.074] \& Plasma membrane [0.096] for P42858 in the pessimistic case, etc., where the square bracketed number denotes probability. The underlined TRUE Labels demonstrates MLMK$T L M$ 's generalization ability rather than misleading tendency. Actually, $M L M K-T L M$ 's misleading tendency is lower than the NT-LHR measures in Table 2 to Table 4 according to the latest Sreiss-Prot database. No training proteins in Hum-mPLoc 2.0 dataset [5] are subjected to the subcellular localization pattern (Nucleus, Cytoplasm, Endoplasmic reticulum, Golgi apparatus, Plasma membrane) as P42858, whereas MLMK-TLM can correctly hit the five labels with different confidence levels, which is hard to achieve by the nearest neighbour based multi-label classifiers [19,28,29,30], because the classifiers assigned to the query protein the labels that belong to the nearest training protein(s). Hum-mPLoc 2.0 web server (http:// www.csbio.sjtu.edu.cn/bioinf/hum-multi-2/) labels O43663, P41222 and P42858 as follows: (1) O43663: Nucleus, without hitting Cytoplasm \& Cytoskeleton; (2) P41222: Endoplasmic reticulum, Golgi apparatus and Nucleus, without hitting Cytoplasm \& Extracell; (3) P42858: Cytoplasm, Golgi apparatus and Nucleus, without hitting Endoplasmic reticulum \& Plasma membrane.

For both the moderate and the pessimistic case, the test proteins' own $G O$ information is removed for the simulation of novel proteins, whereas $M L M K-T L M$ can correctly predicts the test proteins' true labels and underlined TRUE Labels as illustrated in Table 2 to Table 6 . The results show that MLMK-TLM has a good multi-labelling ability for novel multiplex human proteins. From Table 6, we also can see $M L M K-T L M$ shows a certain 
misleading tendency, for example, Nucleus [0.115] \& Plasma membrane [0.181] for P30533 in the optimistic case; Endoplasmic reticulum [0.089] for Q14145 in the moderate case; and Endoplasmic reticulum [0.315] for Q9UHD9 in the pessimistic case, etc. HummPLoc 2.0 web server (http://www.csbio.sjtu.edu.cn/bioinf/hummulti-2/) labels P30533, Q14145 and Q9UHD9 as follows: (1) P30533: Plasma membrane, Endoplasmic reticulum and Extracells, hitting non-target labels Plasma membrane \& Extracells; (2) Q14145: Cytoplasm and Endoplasmic reticulum, hitting non-target label Endoplasmic reticulum; (3) Q9UHD9: Cytoplasm, Endoplasmic reticulum and Nucleus, hitting non-target label Endoplasmic reticulum. Misleading tendency is an important factor that should be given attention for multi-label learning scenario. The advantage of probability outputs is to inform the biologists of the confidence level of each subcellular location, and thus help biologists make a rational decision.

\section{Discussion}

In this paper, we propose a multi-label multi-kernel transfer learning model for human protein subcellular localization (MLMK-TLM), which o further extends our published work $G O-$ $T L M$ and $M K-T L M$ to multi-label learning scenario, such that $M L M K-T L M$ has the following advantages over the existing $G O-$ based models $[2,4,5,14,15,16,17,18,19,20,21,22,23,24,25,26]$ : (1) proper homolog knowledge transfer with rational control over noise from divergent homologs; (2) comprehensive survey of model performance for novel protein; (3) multi-labelling capability with probability interpretation. As compared to single-label learning, multi-label learning is more complicated. In our work, we propose a multi-label confusion matrix and adapt one-againstall multi-class probabilistic outputs to multi-label learning scenario; meanwhile, we formally propose three multi-label learning performance measures: LHR (Label Hit Rate), PLMR (Perfect Label Match Rate) and NT-LHR (Non-target Label Hit Rate). $\mathcal{N T}-L H R$ is formally formulated to measure the model's misleading tendency. The experiments show that MLMK-TLM significantly outperforms the baseline model and demonstrates good multi-labelling ability for novel human proteins. Some findings (predictions) are validated by the latest Swiss-Prot database.

\section{References}

1. Chou KC, Shen HB (2008) Cell-PLoc: A package of Web servers for predicting subcellular localization of proteins in various organisms. Nature Protocols 3: 153-162.

2. Chou KC, Shen HB (2006) Hum-PLoc: A novel ensemble classifier for predicting human protein subcellular localization. Biochemical and Biophysical Research Communications 347: 150-157.

3. Garg A, Bhasin M, Raghava GP (2005) Support vector machine-based method for subcellular localization of human proteins using amino acid compositions, their order, and similarity search. J Biol Chem 280: 14427-14432.

4. Shen HB, Chou KC (2007) Hum-mPLoc: an ensemble classifier for large-scale human protein subcellular location prediction by incorporating samples with multiple sites. Biochem Bio-phys Res Commun 355: 1006-1011.

5. Shen HB, Chou KC (2009) A top-down approach to enhance the power of predicting human protein subcellular localization: Hum-mPLoc 2.0. Analytical Biochemistry 394: 269-274.

6. Hoglund A, Donnes P, Blum T, Adolph H, Kohlbacher O (2006) MultiLoc: prediction of protein subcellular localization using N-terminal targeting sequences, sequence motifs and amino acid composition. Bioinformatics 22(10): 1158-1165

7. Chou KC, Cai YD (2002) Using functional domain composition and support vector machines for prediction of protein subcellular location. J Biol Chem 277: 45765-45769.

8. Mak M, Guo J, Kung S (2008) PairProSVM: protein subcellular localization based on local pairwise profile alignment and SVM. IEEE/ACM Transactions on Computational Biology and Bioinformatics 5(3): 416-422.

9. Pierleoni A, Luigi P, Fariselli P, Casadio R (2006) BaCelLo: a balanced localization predictor. Bioinformatics 22(14): e408-e416.

\section{Supporting Information}

File S1 Full list of perfect label match proteins in the optimistic case. For each multiplex protein in the supplementary documents, there are three lines of description. The first line describes the protein accession; the second line describes the true label(s) of the proteins; and the third line gives the predicted label $(\mathrm{s})$ of the protein. Each predicted label is followed by a squared bracketed number that denotes the probability the protein is predicted to the label.

(DOG)

File S2 Full list of perfect label match proteins in the modest case. The file format dittos.

(DOC)

File S3 Full list of perfect label match proteins in the pessimistic case. The file format dittos. (DOG)

File N S1 Full list of non perfect label match proteins in the optimistic case. The file format dittos. (DOC)

File N S2 Full list of non perfect label match proteins in the modest case. The file format dittos. (DOG)

File N S3 Full list of non perfect label match proteins in the pessimistic case. The file format dittos. (DOC)

\section{Acknowledgments}

Thanks for the anonymous reviewers' helpful advice.

\section{Author Contributions}

Conceived and designed the experiments: SM. Performed the experiments: SM. Analyzed the data: SM. Contributed reagents/materials/analysis tools: SM. Wrote the paper: SM.

10. Mei S, Wang Fei (2010) Amino acid classification based spectrum kernel fusion for protein subnuclear localization. BMC Bioinformatics 11(Suppl 1): S17.

11. Dijk A, Bosch D, Braak C, Krol A, Ham R (2008) Predicting sub-Golgi localization of type II membrane proteins. Bioinformatics 24(16): 1779-1786.

12. Barrell D, Dimmer E, Huntley RP, Binns D, Donovan C, et al. (2009) The GOA database in 2009-an integrated Gene Ontology Annotation resource. Nucleic Acids Research 37: D396-D403.

13. Boeckmann B, et al. (2003) The SWISS-PROT Protein Knowledgebase and Its Supplement TrEMBL. Nucleic Acids Research 31: 365-370.

14. Shen HB, YanqJ, Chou KC (2007) Euk-PLoc: an ensemble classifier for largescale eukaryotic protein subcellular location prediction. Amino Acids 33: 57-67.

15. Shen HB, Chou KC (2010) Virus-mPLoc: A Fusion Classifier for Viral Protein Subcellular Location Prediction by Incorporating Multiple Sites. Journal of Biomolecular Structure \& Dynamics 28: 0739-1102.

16. Chou KC, Cai Y (2003) A new hybrid approach to predict subcellular localization of proteins by incorporating Gene Ontology. Biochem Biophys Res Commun 311: 743-747.

17. Chou KC, Shen HB (2007) Large-Scale Plant Protein Subcellular Location Prediction. Journal of Cellular Biochemistry 100: 665-678.

18. Chou KC, Cai Y (2004) Prediction of protein subcellular locations by GOFunD-PseAA predictor. Biochem Biophys Res Commun 320: 1236-1239.

19. Chou KC, Shen HB (2007) Euk-mPLoc: a fusion classifier for large-scale eukaryotic protein subcellular location prediction by incorporating multiple sites. J Proteome Res 6: 1728-1734.

20. Blum T, Briesemeister S, Kohlbacher O (2009) MultiLoc2: integrating phylogeny and Gene Ontology terms improves subcellular protein localization prediction. BMC Bioinformatics 10: 274. 
21. Tung T, Lee D (2009) A method to improve protein subcellular localization prediction by integrating various biological data sources. BMC Bioinformatics 10(Suppl 1): S43.

22. Lee K, Chuang H, Beyer A, Sung M, Huh W, et al. (2008) Protein networks markedly improve prediction of subcellular localization in multiple eukaryotic species. Nucleic Acids Research 36(20): e136.

23. Huang W, Tunq C, Ho S, Hwang S, Ho S (2008) ProLoc-GO: utilizing informative Gene Ontology terms for sequence-based prediction of protein subcellular localization. BMC Bioinformatics 9: 80.

24. Huang W, Tung C, Huang S, Ho S (2009) Predicting protein subnuclear localization using GO-amino-acid composition features. Biosystems 98(2): 73-9.

25. Mei S, Wang F, Zhou S (2011) Gene ontology based transfer learning for protein subcellular localization. BMC Bioinformatics 12: 44.

26. Mei S (2012) Multi-kernel transfer learning based on Chou's PseAAC formulation for protein submitochondria localization. Journal of Theoretical Biology 293: 121-130.

27. Chou KC, Wu ZC, Xiao X (2011) iLoc-Euk: A Multi-Label Classifier for Predicting the Subcellular Localization of Singleplex and Multiplex Eukaryotic Proteins. PLoS One 6: e18258.

28. Xiao X, Wu ZC, Chou KC (2011) iLoc-Virus: A multi-label learning classifier for identifying the subcellular localization of virus proteins with both single and multiple sites. J Theor Biol 284: 42-51.

29. Xiao X, Wu ZC, Chou KC (2011) A multi-label classifier for predicting the subcellular localization of gram-negative bacterial proteins with both single and multiple sites. PLoS One 6: e20592.

30. Chou KC, Shen HB (2010) Plant-mPLoc: A Top-Down Strategy to Augment the Power for Predicting Plant Protein Subcellular Localization. PLoS ONE 5: e11335.
31. Rajendran L, Knölker H, Simons K (2010) Subcellular targeting strategies for drug design and delivery. Nature Reviews Drug Discovery 9: 29-42.

32. Pan S, Yang Q (2010) A Survey on Transfer Learning. IEEE Transactions on Knowledge and Data Engineering 22(10): 1345-1359.

33. Tu Y, tolovitzky G, Klein U (2002) Quantitative noise analysis for gene expression microarray experiments. PNAS 99(22): 14031-14036.

34. Dai W, Yang Q, Xue G, Yu Y (2007) Boosting for Transfer Learning. Proceedings of the 24th International Conference on Machine Learning.

35. Dai W, Chen Y, Xue G, Yang Q, Yu Y (2008) Translated Learning: Transfer Learning across Different Feature Spaces. Advances in Neural Information Processing Systems (NIPS) 21.

36. Yang Q Chen Y, Xue G, Dai W, Yu Y (2009) Heterogeneous Transfer Learning for Image Clustering via the Social Web. Proceedings of the 47th Annual Meeting of the ACL and the 4th IJCNLP of the AFNLP 2009, pages 19.

37. Altschul S, Madden T, Schaffer A, Zhang J, Zhang Z, et al. (1997) Gapped BLAST and PSI-BLAST: A New Generation of Protein Database Search Programs. Nucleic Acids Research 25: 3389-3402.

38. Wu T, Lin C, Weng R (2004) Probability Estimates for Multi-class Classification by Pairwise Coupling. Journal of Machine Learning Research 5: 975-1005.

39. Shen HB, Chou KC (2010) Gneg-mPLoc: A top-down strategy to enhance the quality of predicting subcellular localization of Gram-negative bacterial proteins. Journal of Theoretical Biology 264: 326-333.

40. Zhu L, Yang J, Shen HB (2009) Multi Label Learning for Prediction of Human Protein Subcellular Localizations. Protein J 28: 384-390.

41. Platt J (1999) Probabilistic outputs for support vector machines and comparison to regularized likelihood methods. In Advances in Large Margin Classifiers. MIT Press. 